\title{
Clinical features of active tuberculosis that developed during anti-tumor necrosis factor therapy in patients with inflammatory bowel disease
}

\author{
Jang Wook Lee ${ }^{1}$, Chang Hwan Choi ${ }^{1}$, Ji Hoon Park ${ }^{1}$, Jeong Wook Kim', Sang Bum Kang ${ }^{2}$, Ja Seol Koo \\ Young-Ho Kim ${ }^{4}$, You Sun Kim ${ }^{5}$, Young Eun Joo ${ }^{6}$, Sae Kyung Chang ${ }^{1}$ \\ Department of Internal Medicine, ${ }^{1}$ Chung-Ang University College of Medicine, Seoul, ${ }^{2}$ The Catholic University of Korea College of Medicine, \\ Daejeon, ${ }^{3}$ Korea University College of Medicine, Seoul, ${ }^{4}$ Sungkyunkwan University School of Medicine, Seoul, Inje University College of \\ Medicine, Seoul, ${ }^{6}$ Chonnam National University Medical School, Gwangju, Korea
}

Background/Aims: Anti-tumor necrosis factor (TNF) therapy for active ulcerative colitis (UC) and Crohn's disease (CD) is associated with increased risks of tuberculosis (TB) infection. We analyzed the incidence and clinical features of Korean patients with inflammatory bowel disease (IBD) who developed active TB during anti-TNF therapy. Methods: Ten cases of active TB developed in patients treated with infliximab $(n=592)$ or adalimumab $(n=229)$ for UC $(n=160)$ or CD $(n=661)$ were reviewed. We analyzed demographics, interval between start of anti-TNF therapy and active TB development, tests for latent TB infection (LTBI), concomitant medications, and the details of diagnosis and treatments for TB. Results: The incidence of active TB was $1.2 \%$ (10/821): $1.5 \%$ (9/592) and $0.4 \%$ (1/229) in patients receiving infliximab and adalimumab, respectively. The median time to the development of active TB after initiation of anti-TNF therapy was three months (range: 2-36). Three patients had past histories of treatment for TB. Positive findings in a TB skin test (TST) and/or interferon gamma releasing assay (IGRA) were observed in three patients, and two of them received anti-TB prophylaxis. Two patients were negative by both TST and IGRA. The most common site of active TB was the lungs, and the active TB was cured in all patients. Conclusions: Active TB can develop during anti-TNF therapy in IBD patients without LTBI, and even in those with histories of TB treatment or LTBI prophylaxis. Physicians should be aware of the potential for TB development during anti-TNF therapy, especially in countries with a high prevalence of TB. (Intest Res 2016;14:146-151)

Key Words: Colitis, ulcerative; Crohn disease; Infliximab; Adalimumab; Tuberculosis

\section{INTRODUCTION}

Tuberculosis (TB) is one of the most serious infectious diseases. In 2013, roughly 9 million new patients were positive for TB worldwide, leading to 1.5 million deaths. TB also ranks highest among all infectious diseases in mortality world-

Received August 18, 2015. Revised March 20, 2016

Accepted March 21, 2016.

Correspondence to Chang Hwan Choi, Department of Internal Medicine, Chung-Ang University College of Medicine, 102 Heukseok-ro, Dongjak-gu, Seoul 06973, Korea. Tel: +82-2-6299-1418, Fax: +82-2-6299-2017, E-mail: gicch@cau.ac.kr

Financial support: None. Conflict of interest: None. wide. ${ }^{1}$ In Korea, about 36 thousand new TB patients were reported in 2013; the nation also has the highest incidence and mortality rates among all countries in the Organization for Economic Cooperation and Development. ${ }^{2}$

$\mathrm{TB}$ is usually transmitted as a cryptogenic infection triggered by the immune defense system of the host, and about $10 \%$ of these manifest themselves as active TB through reactivated infections. Tumor necrosis factor- $\alpha$ (TNF- $\alpha)$ is a cytokine that is central to the immune system's defense against TB infection; ${ }^{3}$ it also contributes to the develop and maintenance of granulomas, which prevent infection. ${ }^{4}$

IBDs, including UC and CD, are chronic inflammatory diseases that affect the entire gastrointestinal tract. They are

\footnotetext{
๑ Copyright 2016. Korean Association for the Study of Intestinal Diseases. All rights reserved.

This is an Open Access article distributed under the terms of the Creative Commons Attribution Non-Commercial License (http://creativecommons.org/licenses/by-nc/4.0)

which permits unrestricted non-commercial use, distribution, and reproduction in any medium, provided the original work is properly cited.
} 
prevalent in Western nations, and their incidence has been increasing steadily in Korea. ${ }^{5}$ Anti-TNF- $\alpha$ antibody is widely used to treat patients with IBD ${ }^{6,7}$ However, adverse effects related to anti-TNF therapy, such as infusion reactions, nervous system abnormalities like demyelinating disease, autoimmune reactions including lupus symptoms, and infections such as TB may develop., ${ }^{8,9}$ Among these, the most significant problem from the clinical perspective is the increased risk of TB. Particularly in Korea, where the prevalence of TB is still high, there is great interest in the occurrence of TB during anti-TNF therapy.

We retrospectively reviewed and analyzed the incidence and clinical features of IBD cases that developed active TB during anti-TNF therapy among patients in 6 academic hospitals.

\section{METHODS}

We distributed questionnaires to 6 academic hospitals in Korea and collected data from active TB cases that developed in patients with IBD during anti-TNF therapy for active inflammation and/or fistula treatments from January 2004 to December 2014. Among patients with CD, over 90\% were treated with infliximab and adalimumab after February 2007 and April 2010, respectively, when their administration for CD was approved by the Korean Food and Drug Administration (KFDA). For patients with UC, infliximab and adalimumab were most used after October 2010 and July 2013, respectively, due to their approval by the KFDA. We investigated the incidence of active $\mathrm{TB}$, as well as the various clinical features, including patient characteristics, periods from initiation of anti-TNF therapy to the diagnosis of TB, tests used to determine latent TB infection (LTBI), concomitant medications, and the details of TB diagnosis and treatments. This study was conducted under the approval of the Clinical Research Ethics Committee of Chung-Ang University Hospital.

\section{RESULTS}

\section{Incidence of Active TB and Patient Characteristics}

Active TB developed in 10 patients with IBD during infliximab $(n=592)$ and adalimumab $(n=229)$ therapy. The incidence of active TB was 1.2\% (10/821): 1.5\% (9/592) and 0.4\% $(1 / 229)$ in patients receiving infliximab with adalimumab, respectively. Their median age at the time of diagnosis of TB was 38 years (range: 19-52), and 8 patients were male $(80.0 \%)$. Analysis of their medical histories revealed that one patient had ankylosing spondylitis and uveitis; another had diabetes, hypertension, and alcoholic liver cirrhosis. Four patients $(40.0 \%)$ had a history of smoking, and 1 continued to smoke even after being diagnosed with TB. The median time from the IBD diagnosis to the start of anti-TNF therapy was 85 months (range: 1-148). Of the 10 patients, 6 had taken corticosteroids and/or azathioprine within 1 month before the development of active TB (Table 1).

Table 1. Baseline Characteristics of the Enrolled Patients

\begin{tabular}{|c|c|}
\hline Characteristic & Value, no. \\
\hline Age, median (range), yr & $38(19-52)$ \\
\hline \multicolumn{2}{|l|}{ Sex } \\
\hline Male & 8 \\
\hline Female & 2 \\
\hline BMI, median (range) & $19.0(13.8-28.4)$ \\
\hline \multicolumn{2}{|l|}{ Disease phenotype - CD (n=9) } \\
\hline \multicolumn{2}{|l|}{ Location } \\
\hline lleal & 1 \\
\hline Colonic & 1 \\
\hline Ileocolonic & 7 \\
\hline \multicolumn{2}{|l|}{ Complications } \\
\hline Strictures & 5 \\
\hline Intraabdominal abscess & 2 \\
\hline \multicolumn{2}{|l|}{ History of operations related to CD } \\
\hline Right hemicolectomy/cecectomy & 1 \\
\hline Small bowel resection & 1 \\
\hline Anal fistulotomy & 3 \\
\hline \multicolumn{2}{|l|}{ Disease phenotype - UC ( $n=1)$} \\
\hline Proctitis & 1 \\
\hline \multicolumn{2}{|l|}{ Indication for anti-TNF therapy } \\
\hline \multicolumn{2}{|l|}{$\mathrm{CD}$} \\
\hline Active luminal disease & 6 \\
\hline Active luminal and fistulizing disease & 3 \\
\hline \multicolumn{2}{|l|}{ UC } \\
\hline Steroid dependent disease & 1 \\
\hline \multicolumn{2}{|l|}{ Biologic agents } \\
\hline Infliximab & 9 \\
\hline Adalimumab & 1 \\
\hline \multicolumn{2}{|l|}{ Concomitant medications* } \\
\hline Corticosteroids & 3 \\
\hline Azathioprine & 4 \\
\hline Azathioprine plus corticosteroids & 1 \\
\hline 5-aminosalicylic acid & 5 \\
\hline
\end{tabular}

TNF, tumor necrosis factor.

*One or more drugs could be used. 


\section{Previous Active TB History and Latent TB}

Three patients had histories of previously active TB, which was cured with anti-TB medications. Three patients had Bacillus Calmette-Guerin vaccine cicatrices, 2 did not, and the rest were not examined. A tuberculin skin test (TST) and/or interferon gamma releasing assay (IGRA) were performed in 9 patients to screen for LTBI before anti-TNF treatment. Six patients were examined by TST, 7 by IGRA, and 4 by both methods. They all underwent chest X-ray examinations to screen for pulmonary TB. One patient did not have

Table 2. Results of Screening for Latent Tuberculosis

\begin{tabular}{lc}
\multicolumn{1}{c}{ Screening test } & Value, no. \\
\hline Tuberculin skin test & 2 \\
Positive & 4 \\
Negative & 4 \\
Not done & \\
IGRA test & 1 \\
Positive & 6 \\
Negative & 3 \\
Not done & \\
Chest X-ray & 1 \\
Suggestive of healed tuberculosis & 9 \\
Normal & \\
\hline
\end{tabular}

IGRA, interferon gamma releasing assay. screening tests for LTBI due to previous treatment history of TB. The TST was positive in 2 patients, while the IGRA was positive in 1 patient (Table 2). Two of the three patients were treated for LTBI with the combined therapy of isoniazid (300 $\mathrm{mg}$ ) and rifampin $(600 \mathrm{mg})$. Detailed clinical findings of the TB infections in all enrolled patients are listed in Table 3.

\section{Diagnosis of Active TB}

The median time between the start of anti-TNF therapy and TB occurrence was 3 months (range: 2-36) (Table 4). Of the 2 patients who received LTBI treatments, 1 developed active TB after 61 days of treatment with isoniazid and rifampin, and had started infliximab 11 days after beginning the anti-TB medications. In the second patient, infliximab was started after isoniazid and rifampin had been administered for 3 months, and active TB developed 18 months later.

Active TB was diagnosed by AFB staining for mycobacteria from tissue or sputum samples $(\mathrm{n}=7)$, AFB culture $(\mathrm{n}=3)$, TB PCR assay ( $\mathrm{n}=2)$, or clinically with chest X-rays and CT scans of the thorax and abdomen $(n=3)$. All cases of active TB involved the lungs. Two patients had also extra-pulmonary TB on the mediastinal lymph nodes and peritoneum, respectively. Cough was the most common symptom, followed by fever and sputum (Table 4). Fig. 1 shows a representative chest X-ray for the active TB developed in patient with CD.

Table 3. Clinical Characteristics of Patients With Active Tuberculosis (TB) Infections

\begin{tabular}{|c|c|c|c|c|c|c|c|c|c|}
\hline No. & $\begin{array}{c}\text { Patient } \\
\text { (age } / \text { sex) }\end{array}$ & TB history & $\mathrm{TST}^{\mathrm{b}}$ & IGRA & Chest X-ray & $\begin{array}{l}\text { LTBI treatment } \\
\text { (duration, mo) }\end{array}$ & IMMs & Steroids & $\begin{array}{l}\text { Interval to TB } \\
\text { infection (mo) }\end{array}$ \\
\hline 1 & $38 / M$ & Yes/Lung & ND & ND & Normal & ND & No & Yes & 3 \\
\hline 2 & $32 / M$ & No & $(-)$ & $(-)$ & Normal & ND & Yes & No & 3 \\
\hline 3 & $41 / \mathrm{M}$ & No & ND & $(-)$ & Normal & ND & No & No & 2 \\
\hline 4 & $25 / M$ & No & ND & $(-)$ & Normal & ND & Yes & No & 3 \\
\hline 5 & $28 / F$ & No & $(-)$ & ND & Normal & ND & No & No & 12 \\
\hline 6 & $43 / \mathrm{M}$ & $\begin{array}{l}\text { Yes/Lung \& } \\
\text { peritoneum }\end{array}$ & ND & $(+)$ & Normal & INH+RFP (3) & No & No & 18 \\
\hline 7 & $52 / \mathrm{M}$ & No & $(+)$ & $(-)$ & Normal & INH+RFP (3) & Yes & Yes & 2 \\
\hline 8 & 19/M & No & $(-)$ & ND & Normal & ND & No & No & 6 \\
\hline 9 & $40 / F$ & Yes/Lung & $(-)$ & $(-)$ & TB scar & ND & No & Yes & 2 \\
\hline 10 & $39 / \mathrm{M}$ & No & $(+)$ & $(-)$ & Normal & ND & Yes & No & 36 \\
\hline
\end{tabular}

${ }^{\mathrm{a}}$ Age at diagnosis of TB.

${ }^{b} A$ purified protein derivative reaction measuring less than $10 \mathrm{~mm}$ was considered negative for the TST.

TST, tuberculosis skin test; IGRA, interferon gamma releasing assay; LTBI, latent tuberculosis infection; IMM, immunomodulator; M, male; F, female; ND, not done; INH, isoniazid; RFP, rifampin. 
Table 4. Diagnosis of Active Tuberculosis (TB)

\begin{tabular}{lc}
\multicolumn{1}{c}{ Variable } & Value, no. \\
\hline $\begin{array}{l}\text { Interval between first dose and diagnosis of TB, median } \\
\text { (range) mo. }\end{array}$ & $3(2-36)$ \\
Method of diagnosis* & \\
AFB stain & 7 \\
Culture & 3 \\
PCR assay & 2 \\
Clinical diagnosis & 3 \\
Disease involvement & \\
Lung alone & 8 \\
Lung and mediastinal lymph nodes & 1 \\
Lung and peritoneum & 1 \\
Symptoms* & \\
Fever ( $>38.0^{\circ} \mathrm{C}$ ) & 5 \\
Cough & 8 \\
Sputum & 4 \\
Others (myalgia, dyspnea, chest pain, abdominal \\
discomfort)
\end{tabular}

${ }^{*}$ One or more varible are possible.

\section{Anti-TB Treatments}

Among all patients included in this study, 9 were treated with isoniazid, ethambutol, rifampin, and pyrazinamide (HERZ) for more than 6 months immediately after the diagnosis of active TB. The other patient was administered cycloserine, prothionamide, and levofloxacin for 20 months after 1 month of HERZ medication, which was discontinued due to the side effects. The median duration of anti-TB treatment was 7 months (range: 6-21), and the active TB was cured in all patients.

\section{Treatments for IBD After TB Treatment}

Three patients did not restart treatment with the anti-TNF agents after completing the anti-TB treatments. The remaining 7 patients continued $(n=1)$ or restarted $(n=6)$ anti-TNF therapy. Three of the restarted patients were administered anti-TNF agent upon completion of the anti-TB treatments, while anti-TNF therapy was started before the completion of the anti-TB treatments in the other 3 patients. One patient continued anti-TNF therapy even after the diagnosis of active TB. Among the patients who continued or restarted antiTNF therapy, no cases of TB recurred.

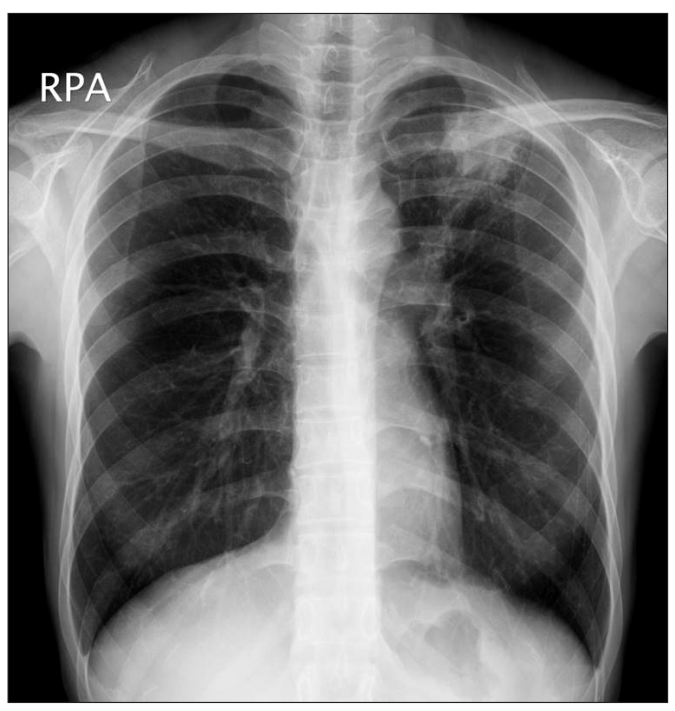

Fig. 1. Chest $X$-ray findings. A representative chest $X$-ray for the active tuberculosis developed in patients with $C D$ during anti-tumor necrosis factor therapy. A patchy heterogeneous consolidation is visible in the left upper lobe of the lung.

\section{DISCUSSION}

Since the first use of biologic agents in Korea in 2001, antiTNF therapy has played an important role in treating various chronic inflammatory diseases, including rheumatoid arthritis, ankylosing spondylitis, psoriasis, and psoriatic arthritis. However, recent reports have shown that the risks of TB may increase from 1.6 to 25.1 -fold, ${ }^{10}$ thus it is mandatory to screen for TB when administering anti-TNF agents and to prescribe anti-TB medication when patients are diagnosed with LTBI. The incidence of active TB during anti-TNF therapy in Korean patients with rheumatic diseases, including rheumatoid arthritis and ankylosing spondylitis, is $1.0 \%-1.7 \% .{ }^{11-14} \mathrm{~A}$ recent study in South Korea conducted with 873 IBD patients who had undergone anti-TNF therapy reported 25 cases (2.9\%) of active TB. ${ }^{15}$ In contrast, the incidence in Western countries is $0.1 \%-0.4 \%$, a much lower rate than that reported in Korea. ${ }^{16,17}$ In our study, the incidence of active TB developed during anti-TNF therapy in patients with IBD was high (1.2\%), similar to the incidences reported in other Korean studies. Active TB developed even in patients who had received antiTB treatments for previous active TB occurrences as well as those who had taken anti-TB medications for LTBI. The most common site of active TB was the lung, and the time interval to the development of active TB from the initiation of antiTNF therapy was generally short. The symptoms, diagnosis, and response to treatments were not different from those of conventional TB, although our study patients were on a spe- 
cific situation of anti-TNF therapy. ${ }^{18}$

According to the Korean guidelines for TB, patients who are to be treated with anti-TNF agents should first be checked for TB history, contact history with patients with $\mathrm{TB}$, present suspected symptoms of TB, and should undergo a chest X-ray examination. ${ }^{2,19}$ In addition, screening tests for LTBI should be used in accordance with criteria developed for immunocompromised patients using IGRA alone or with an integrated test including IGRA and TST. TST alone is not recommended for testing for LTBI. The integrated test considers patients positive for LTBI if either of the two tests has a positive result; patients are also diagnosed if there is a naturally treated TB lesion on chest X-ray without any anti-TB treatment history, regardless of any LTBI screening test result. Among the patients included in our study, LTBI screening was not conducted systematically. The screening tests were performed on 9 patients among the 10 cases, and 3 had positive TST or IGRA results. Among the 6 patients tested by TST, 2 were not tested by IGRA; among the 7 patients who were tested by IGRA, three were not tested by TST. Both the TST and IGRA show sensitivities of about $80 \%$ in screening for LTBI and are known to show lower sensitivities under immunosuppressive conditions..$^{20}$ In our study, 6 cases used steroids and/or immunomodulators concomitantly. Therefore, the TST or IGRA results might have resulted in false negatives, especially in patients who were not simultaneously assessed using the other test. ${ }^{21,22}$ Accordingly, the Korean guidelines for TB recommend an integrated test incorporating both IGRA and TST to screen for LTBI. ${ }^{2,19}$ Thus, more aggressive screening is necessary to prevent reactivation of latent TB. However, the usefulness of the tests for LTBI should be validated, because active TB can develop in patients with negative LTBI test findings.

Of the 2 patients diagnosed with active TB despite having completed latent TB treatments, 1 started anti-TNF treatment 11 days after beginning treatment for latent TB. Following LTBI diagnosis, the Korean guidelines for TB recommend initiation of anti-TNF therapy 3 weeks after prescription of anti-TB medications; however, they also mention that antiTNF therapy may be started early in some cases. ${ }^{2,19}$ Our case followed this consideration rather than the standard recommendations, indicating the need for further study of these considerations.

Keane et al. ${ }^{23}$ reported a median interval of 3 months in 70 patients with active TB after the start of infliximab injections; $75 \%$ of the patients were diagnosed within 5.5 months. Similarly, 7 patients (70\%) in our study were diagnosed with active TB within 6 months from the start of anti-TNF therapy.
However, recent two Korean studies with IBD patients reported relatively long intervals between the start of anti-TNF treatment and diagnosis of active TB: median 23 months (range: 2-76) and 7 months (range: 2-33), respectively. ${ }^{15,24}$ We did not know the exact reason for this discrepancy. However, one plausible explanation is that our study might have included mainly cases of LTBI reactivation, while the other Korean studies included more cases of de novo TB infection. Since South Korea is a moderate-risk country for TB, patients could be newly exposed to tubercular bacillus and thus became infected in the later periods. Collectively, the risk of TB development has to be considered in both the later as well as the early periods of anti-TNF therapy in patients with IBD.

Only two of the TB cases in our study were affected in sites other than the lungs, a finding similar to the frequency of extra-pulmonary TB cases (20.0\%) among all reported patients with TB in 2014. ${ }^{25}$ Although direct comparison is difficult, as the total number of patients of our study was too small, the frequency of extra-pulmonary TB was much smaller than the 57\%-75\% reported in Western countries. ${ }^{23,26,27}$ A recent Korean study with IBD patients also reported that the proportion of extra-pulmonary TB was 16\% (4 of 25 cases). ${ }^{15}$ However, another Korean study suggested a high incidence of extrapulmonary TB of $81.3 \%$ ( 13 of 16 cases). ${ }^{24}$ Although we also do not know the exact reasons for the different manifestation, this discrepancy might be due to differences in study populations, such as variations in the regional prevalence of LTBI. Further studies are needed to clarify the common involved site of TB and its association with anti-TNF therapy and prevalence of $\mathrm{TB}$.

In conclusion, the development of active $\mathrm{TB}$ is not rare in IBD patients undergoing anti-TNF therapy, and de novo TB infections as well as the reactivation of latent TB can occur, especially in countries with a high prevalence of TB. In addition, active TB can develop even in patients who had received anti-TB treatments for previous active $\mathrm{TB}$ and who had taken anti-TB medications for LTBI. Physicians should thoroughly review patient histories, chest X-ray findings, and screen for LTBI before starting anti-TNF therapy, and should be aware of the potential for TB development throughout all periods of anti-TNF therapy.

\section{REFERENCES}

1. World Health Organization. Global tuberculosis report 2014. Geneva: World Health Organization, 2014. 
2. Joint Committee for the Revision of Korean Guidelines for Tuberculosis; Korea Centers for Disease Control and Prevention. Korean guidelines for tuberculosis. 2nd ed. Cheongju: Korea Centers for Disease Control and Prevention, 2014.

3. Serbina NV, Flynn JL. Early emergence of $\mathrm{CD}^{+} \mathrm{T}$ cells primed for production of type 1 cytokines in the lungs of Mycobacterium tuberculosis-infected mice. Infect Immun 1999;67:3980-3988.

4. Flynn JL, Chan J. Immunology of tuberculosis. Annu Rev Immunol 2001;19:93-129.

5. Thia KT, Loftus EV, Jr., Sandborn WJ, Yang SK. An update on the epidemiology of inflammatory bowel disease in Asia. Am J Gastroenterol 2008;103:3167-3182.

6. Lee KM, Jeen YT, Cho JY, et al. Efficacy, safety, and predictors of response to infliximab therapy for ulcerative colitis: a Korean multicenter retrospective study. J Gastroenterol Hepatol 2013;28:1829-1833.

7. Kim YJ, Kim JW, Lee CK, et al. Clinical outcome of treatment with infliximab in Crohn's disease: a single-center experience. Korean J Gastroenterol 2013;61:270-278.

8. Cush JJ. Safety overview of new disease-modifying antirheumatic drugs. Rheum Dis Clin North Am 2004;30:237-255.

9. Imperato AK, Bingham CO 3rd, Abramson SB. Overview of benefit/risk of biological agents. Clin Exp Rheumatol 2004;22(5 Suppl 35):S108-S114.

10. Solovic I, Sester M, Gomez-Reino JJ, et al. The risk of tuberculosis related to tumour necrosis factor antagonist therapies: a TBNET consensus statement. Eur Respir J 2010;36:1185-1206.

11. Chung KB, Lee EY, Im JP, Han SK, Yim JJ. Clinical characteristics and treatment responses of patients who developed tuberculosis following use of a tumor necrosis factor- $\alpha$ inhibitor. Korean J Intern Med 2013;28:174-179.

12. Seong SS, Choi CB, Woo JH, et al. Incidence of tuberculosis in Korean patients with rheumatoid arthritis (RA): effects of RA itself and of tumor necrosis factor blockers. J Rheumatol 2007;34:706-711.

13. Suh YS, Kwok SK, Ju JH, Park KS, Park SH, Yoon CH. Safe readministration of tumor necrosis factor-alpha (TNFalpha) inhibitors in patients with rheumatoid arthritis or ankylosing spondylitis who developed active tuberculosis on previous antiTNF $\alpha$ therapy. J Korean Med Sci 2014;29:38-42.

14. Yoo IK, Choung RS, Hyun JJ, et al. Incidences of serious infections and tuberculosis among patients receiving anti-tumor necrosis factor-alpha therapy. Yonsei Med J 2014;55:442-448.

15. Byun JM, Lee CK, Rhee SY, et al. Risks for opportunistic tuberculosis infection in a cohort of 873 patients with inflammatory bowel disease receiving a tumor necrosis factor-alpha inhibitor. Scand J Gastroenterol 2015;50:312-320.
16. Dixon WG, Hyrich KL, Watson KD, et al. Drug-specific risk of tuberculosis in patients with rheumatoid arthritis treated with anti-TNF therapy: results from the British Society for Rheumatology Biologics Register (BSRBR). Ann Rheum Dis 2010;69:522528.

17. Tubach F, Salmon D, Ravaud P, et al. Risk of tuberculosis is higher with anti-tumor necrosis factor monoclonal antibody therapy than with soluble tumor necrosis factor receptor therapy: The three-year prospective French Research Axed on Tolerance of Biotherapies registry. Arthritis Rheum 2009;60:18841894.

18. Kwon YS, Koh WJ. Diagnosis of pulmonary tuberculosis and nontuberculous mycobacterial lung disease in Korea. Tuberc Respir Dis (Seoul) 2014;77:1-5.

19. Shim TS. Diagnosis and treatment of latent tuberculosis infection in patients with inflammatory bowel diseases due to initiation of anti-tumor necrosis factor therapy. Intest Res 2014;12:12-19.

20. Pai M, Denkinger CM, Kik SV, et al. Gamma interferon release assays for detection of Mycobacterium tuberculosis infection. Clin Microbiol Rev 2014;27:3-20.

21. Holden M, Dubin MR, Diamond PH. Frequency of negative intermediate-strength tuberculin sensitivity in patients with active tuberculosis. N Engl J Med 1971;285:1506-1509.

22. Diagnostic Standards and Classification of Tuberculosis in Adults and Children. This official statement of the American Thoracic Society and the Centers for Disease Control and Prevention was adopted by the ATS Board of Directors, July 1999. This statement was endorsed by the Council of the Infectious Disease Society of America, September 1999. Am J Respir Crit Care Med 2000;161:1376-1395.

23. Keane J, Gershon S, Wise RP, et al. Tuberculosis associated with infliximab, a tumor necrosis factor alpha-neutralizing agent. N Engl J Med 2001;345:1098-1104.

24. Kim ES, Song GA, Cho KB, et al. Significant risk and associated factors of active tuberculosis infection in Korean patients with inflammatory bowel disease using anti-TNF agents. World J Gastroenterol 2015;21:3308-3316.

25. Ko WY, Shon HJ, Lee HK, et al. Annual Report on the Notified Tuberculosis in Korea 2014, Korea Centers for Disease Control and Prevention, 2015.

26. Abreu C, Magro F, Santos-Antunes J, et al. Tuberculosis in antiTNF- $\alpha$ treated patients remains a problem in countries with an intermediate incidence: analysis of 25 patients matched with a control population. J Crohns Colitis 2013;7:e486-492.

27. Wolfe F, Michaud K, Anderson J, Urbansky K. Tuberculosis infection in patients with rheumatoid arthritis and the effect of infliximab therapy. Arthritis Rheum 2004;50:372-379. 